\title{
Review and Synthesis of Smoking Cessation Interventions in The Canadian Aboriginal Population
}

\author{
Mamdouh M. Shubair \\ School of Health Sciences, University of Northern British Columbia, 3333 University Way, Prince George, BC, V2N 4Z9; \\ *Corresponding Author:mamdouh.shubair@unbc.ca
}

Copyright (c) 2014 Horizon Research Publishing All rights reserved.

\begin{abstract}
Smoking is a major public health concern among the Aboriginal Canadian Population. The age-adjusted smoking-attributable death rates are two times those of other Canadians; $20 \%$ of adult deaths among Aboriginal Canadians is attributable to smoking. There is no research that implemented and examined interventions of smoking cessation in Aboriginal Canadians, particularly in Northern British Columbia. This paper represents a critical review of some of the successful smoking cessation programs conducted in different jurisdictions. It is evident that little consideration is given to Aboriginal knowledge transfer in program development and delivery. This review indicates that in order for an Aboriginal cessation strategy to be successful, culturally relevant criteria must be identified and implemented. Without consideration of culture, there will always be limited success in tobacco cessation initiatives. To be effective, smoking cessation programs need to be comprehensive. Social determinants of health and socio-economic disparities that influence health status outcomes need to be considered as they have major influence in adopting smoking among Canadian Aboriginals. These factors have implications for public health policy.
\end{abstract}

Keywords Smoking Cessation; Culture; Socio-Economic Disparities; Community-Based Participatory Research; Aboriginal Canadians; Social Determinants of Health

\section{Context}

The Northern Cancer Control Strategy (NCCS) aims to enhance cancer control outcomes in Northern BC through a collaborative approach with the Ministry of Health Services, Provincial Health Services Authority (PHSA), BC Cancer Agency (BCCA) and the Northern Health authority $(\mathrm{NH})$. The strategy addresses the full spectrum of cancer care and is designed to enhance and augment existing programs already in place in the North. Additionally, as stated in the Business Plan, the strategy will add new program elements that will fully integrate the NCCS with the current efforts of the
BCCA in other regional centres [1].

The NCCS includes program enhancements for the complete continuum of cancer care. There are six core projects that include prevention and promotion, screening, detection and diagnosis, treatment, psychosocial care and palliative/end of life care. Additionally, there are seven enabling platforms that include enhanced information technology, telemedicine, navigation, primary care, human resources, monitoring, research and evaluation and Aboriginal health. The enabling platforms must be developed and support the core projects for the strategy to be a success. A four year capital project is another significant component of the NCCS and will create a new cancer centre in Prince George. This state of the art BCCA facility will be the sixth in the province, and the first in the North. The BCCA Centre for the North will form the hub of the NCCS, providing the radiation therapy and systemic therapy available in other regional centres and supporting the network of cancer control initiatives throughout the North [1].

In response to the NCCS goal of enhancing the clinical components of cancer care, a review of national smoking cessation initiatives was reviewed to encourage a more thorough approach to working with the Aboriginal Population in Northern BC. Identification of culturally relevant programs strengthens existing initiatives and ensures there is no duplication of efforts by building upon lessons learned from previously developed programs.

Northern BC has the largest population of Aboriginal people in the province and due to geographic, social and cultural diversity within and between communities, it is essential to assess the specific health needs of community members, to determine concerns about access to cancer care and to establish a baseline of existing data that will help inform the strategy. One area that has been identified by communities and agencies alike is the need to develop and implement culturally appropriate and environmentally relevant intervention programs for tobacco cessation. To this end, a critical review of national programs was undertaken to determine effective cessation programs across different jurisdictions that helped individuals quit smoking. This review provided the opportunity to identify gaps in service 
delivery and to help inform culturally appropriate program development. This review supports the holistic world view of the Aboriginal Population.

\section{Methods}

The literature for this review was gathered through searches of the scientific databases Medline Ovid, ISI Web of Science, and Cochrane Reviews, plus stakeholder provision of grey literature and various reports. The keywords and medical subject headings (MeSH) that provided the most useful information were: 'smoking cessation', 'intervention', 'best practices', 'public health', and 'Aboriginal/First Nations/Native American/American Indian'. Relevancy testing consisted of reading of titles and abstracts of articles to find appropriate studies while excluding duplicates. Inclusion criteria for papers entailed if they were peer-reviewed English language literature, randomized controlled trials, observational studies, or systematic reviews, related to smoking cessation and public health interventions. We focused on papers that were Canadian, American, and Australian in origin.

\section{Results}

The Aboriginal peoples of Canada comprise several groups, including First Nations, Métis, and Inuit. The rates of tobacco use are higher among the First Nations Population than among other Canadians [2]. Among the leading causes of death for Aboriginal adults are chronic diseases associated with cigarette smoking, and the highest reported medical conditions for Aboriginal youth are also associated with tobacco use [3]. There is paucity of research which examined community-based interventions for smoking cessation among Aboriginals, particularly in the province of British Columbia and Canada. The review highlights which programs have demonstrated success in reducing smoking cessation rates and which types of interventions have been unsuccessful or are not recommended for the Aboriginal Population.

A number of smoking cessation interventions were examined which included counselling plus group interventions, individual interventions, self-help, mass media and clinical aids. Specific programs were reviewed regarding Aboriginal versus non-Aboriginal uptake of programs. As an overview, group therapeutic interventions were considered to have limited success among the Aboriginal Population as individual counselling is considered to be more culturally appropriate and effective when combined with nicotine replacement therapy (NRT) [4]. Self-help behavioural interventions were no more effective than brief personal advice [5]. Alternatively, Aboriginal-specific media campaigns were more likely to have greater impact than a general mass media campaign [4]. Clinical aids for health care professionals provided enhanced participation in cessation interventions by having chart reminders on patients' medical charts to prompt reminders to the provider to routinely discuss tobacco cessation.

Specific cessation programs reviewed found mixed results. Two examples are discussed here. SmokeCheck is a brief intervention program that has potential to make a positive impact on decreasing Aboriginal smoking rates. If the full capacity of the SmokeCheck project is to be realized, it is essential that it be integrated into the routine practice of all health care staff providing primary health care to Aboriginal clients [6]. Review of this initiative, however, indicated that not all health care staff considered this approach in their service delivery. Likewise, Give up the Smokes program reported a 30\% quit rate for their Aboriginal clients [7]. The increased quit rate may be due to the provision of free NRT to those attending the required course and the high level of interactive and visually stimulating activities such as Aboriginal artwork use.

Quitlines were found to be a cost-effective method at reaching a large proportion of Aboriginal smokers; they were judged to be effective at helping Aboriginal smokers quit. Little consideration was given however to identifying the challenges of reaching Aboriginal smokers who live in rural and remote areas.

In summary, there is a strong dose-response relationship between the frequency and intensity of tobacco dependence counselling and its effectiveness [8]. Likewise, pharmacotherapy is found to be an essential element of smoking cessation interventions while incentive-based cessation programs have shown moderate success [9]. Finally, it is recognized that although clinical interventions are moderately successful, improving cessation rates of heavy smokers may require new clinical programs and a review of public health and social policy programs to identify an appropriate community-based and holistic health approach in counselling Aboriginal clients.

\section{Discussion}

One of the most important elements of these data is that community aspects of cessation and metrics of success are quite different than the clinically-based model of cessation. Many of the programs reviewed do not focus on the 'community' and how the community views a successful cessation initiative. The question of what constitutes 'Best Practice' for cessation programs among Canadian Aboriginal communities remains largely unanswered.

Mitchell (2007) states that components for a successful Aboriginal cessation strategy must include the following: cultural relevance, local orientation and facilitation, flexibility and responsiveness, facilitator training, holistic, highlight traditional activities, knowledge and values, recognize contemporary lifestyles, cooperate with existing systems and resources and recognize partnerships for strength and improved success. In order for these components to be developed and successfully implemented, 
knowledge transfer must be incorporated. Knowledge transfer in an Aboriginal context can be described as the "transfer between Indigenous knowledge and Western science" [10]. Likewise, the site of transfer is the small area of overlap between Western and Indigenous ontology (what constitutes reality) and epistemology (how we know what we know) [10].

The current author denotes that the focus of many tobacco cessation initiatives is on clinical components of care with little consideration given to culturally appropriate components of cessation. In order for cessation programs to be successful in Aboriginal communities, we must recognize the comprehensive components of a successful initiative as being grounded in the community and supported by the community leaders who need to be 'champions'. Too often, program development is neither culturally relevant nor all-inclusive to encompass all essential elements of a comprehensive tobacco cessation program, as it may have been developed outside the community. As a result, the initiative will not be locally oriented and facilitated. Likewise, cessation programs must be flexible and responsive to the needs of the community in order to have support from community members. Capacity building and empowerment within communities is pivotal to successful program implementation. Trained community counsellors will ensure that a tobacco cessation initiative is holistic and incorporates traditional activities, knowledge and values while recognizing that all Aboriginal people do not lead traditional lifestyles. Identifying and incorporating an activity that has both traditional and western components and is of interest to community members is more likely to be successful if identified by the community members themselves. Working with existing systems and developing relationships and partnerships with the community will empower the community and promote program success. Until culturally appropriate and holistic aspects of cessation initiatives are considered there will always be limited success with Aboriginal tobacco cessation efforts.

There are other factors (determinants) that are critical to the success of designing smoking cessation interventions for Aboriginal communities, and need to be taken into account. These include tackling the socioeconomic disparities that Aboriginal Canadians face overall, compared with the rest of Canadians. We argue that major issues of unemployment, poor housing conditions, homelessness, low education attainment levels, income inequality, and inequity in access to health care need to be addressed from an upstream social policy and public health perspective. As noted by several researchers, these social determinants of health affect lifestyle choices/behaviours such as smoking and impact health outcomes such as cancer and cardiovascular disease [11-13].

Last but not least, a central tenet of tobacco control policies and programs is their need to be comprehensive. Tobacco cessation interventions and tobacco control strategies will only ever work modestly well, and only in the context of a comprehensive program [14]. Many elements of a comprehensive tobacco cessation protocol are missing in Canada's Aboriginal communities. First Nations people have access to tax-free cigarettes. In many Canadian Aboriginal communities, there continues to be exposure to environmental tobacco smoke (ETS), or second-hand smoke, in workplace settings and in public places when it has been brought to an end in all other Canadian communities. Tobacco trade, including tobacco advertising, continues to thrive in many Aboriginal communities and cigarettes are sold without any graphic labels or health warnings

\section{Conclusions}

To conclude, without consideration of culture, there will always be limited success in tobacco cessation initiatives. Many key elements of comprehensive tobacco control strategies are lacking in the Canadian Aboriginal Population. There are significant socio-economic disparities faced by Aboriginal Canadians which have major influence in their adoption of smoking behaviour. Until these multifaceted gaps are addressed, the current author believes that no smoking cessation program, whether culturally appropriate or not, is likely to be very effective. These gaps and disparities have implications for public health and social policy; therefore, further community-based participatory research (CBPR) and population-based interventions in the Canadian Aboriginal Population are warranted.

\section{REFERENCES}

[1] The Northern Cancer Control Strategy Business Plan (2010). A Joint Initiative between Northern Health and the PHSA/BC Cancer Agency.

[2] Wardman, A.E.D. and Khan, N.A. (2004). Tobacco cessation pharmacotherapy use among First Nations persons residing within British Columbia. Nicotine \& Tobacco Research; 6(4): 689-692.

[3] Hutchinson, P.J., Richardson, C.G., \& Bottorff, J. L. (2008). Emergent Cigarette Smoking, Correlations with Depression and Interest in Cessation among Aboriginal Adolescents in British Columbia. Canadian Journal of Public Health; 99(5): 418-422.

[4] Power, J., Grealy, C., \& Rintoul, D. (2009). Tobacco interventions for Indigenous Australians: A review of current evidence. Health Promotion Journal of Australia; 20(3): 186-194.

[5] Lancaster, T. \& Stead, L.F. (2009). Self-help interventions for smoking cessation. Cochrane Database of Systematic Reviews; 2: 1-90. Available from: http://www.thecochranelibrary.com/userfiles/ccoch/file/Worl d\%20No\%20Tobacco\%20Day/CD001118.pdf

[6] Hearn, S., Rose, M., Wise, M., Nancarrow, H., Bauman, A., Massi, L., \& Conigrave, K. (2010). The NSW SmokeCheck Aboriginal Tobacco Prevention Project 
2007-2008. Centre for Health Advancement, Australia.

[7] Gould, G.S., McGechan, A., \& van der Zwan, R. (2010). Give up the smokes: a smoking cessation program for Indigenous Australians. 10th national Rural Health Conference July 2010.

[8] Anderson, J. E., Jorenby, D. E., Scott, W. J., \& Fiore, M. C. (2002). Treating Tobacco Use and Dependence: An Evidence-Based Clinical Practice Guideline for Tobacco Cessation. CHEST; 121(3): 932-941.

[9] Robles, G.I., Singh-Franco, D., \& Ghin, H. L. (2008). A review of the efficacy of smoking cessation pharmacotherapies in nonwhite populations. Clinical Therapy; 30(5): 800-812.

[10] Mitchell, S. (2007). Tobacco Cessation Strategies for First Nations, Inuit and Métis: An environmental scan and annotated bibliography. Aboriginal Act Now 2007.
[11] Marmot, M., \& Wilkinson, R.G. [editors]. Social Determinants of Health. Second Edition. London: Oxford University Press; 2006.

[12] Reid, J. L., Hammond, D., \& Driezen, P. (2010). Socio-economic status and smoking in Canada, 1999-2006: Has there been any progress on disparities in tobacco use? Canadian Journal of Public Health; 101(1): 73-78.

[13] Scollo, M.M., \& Winstanley, M. H. [editors]. Tobacco in Australia: Facts and Issues. Third Edition. Melbourne: Cancer Council Victoria; 2008. Available from: http://www.tobaccoinaustralia.org.au (Accessed May 18, 2011).

[14] Greaves, L., Johnson, J., Bottorff, J., Kirkland, S., Jategaonkar, N., McGowan, M., McCullough, L., \& Battersby, L. (2006). What are the effects of tobacco policies on vulnerable populations? A better practices review. Canadian Journal of Public Health; 97(4): 310-315. 